\title{
Insight into the genetic architecture of back pain and its risk factors from a study of 509,000 individuals
}

Maxim B Freidin ${ }^{1} *$, Yakov A Tsepilov ${ }^{2,3,4} *$, Melody Palmer ${ }^{5}$, Lennart C Karssen ${ }^{4}$, CHARGE

Musculoskeletal Working Group, Pradeep Suri ${ }^{6,7,8}$, Yurii S Aulchenko ${ }^{4}$, Frances MK Williams ${ }^{1 \#}$

1 - Department of Twin Research and Genetic Epidemiology, School of Life Course Sciences, King's College London, London, UK;

2 - Novosibirsk State University, Faculty of Natural Sciences, Novosibirsk, Russia;

3 - Institute of Cytology and Genetics, Siberian Branch of Russian Academy of Sciences, Novosibirsk, Russia;

4 - PolyOmica, 's-Hertogenbosch, The Netherlands;

5 - Department of Medical Genetics, University of Washington, Seattle, USA;

6 - Seattle Epidemiologic Research and Information Center (ERIC), Veterans Affairs Office of Research and Development, Seattle, USA;

7 - Rehabilitation Care Services, VA Puget Sound Healthcare System, Seattle, USA.

8 - Department of Rehabilitation Medicine, University of Washington, Seattle, USA

*These authors contributed equally to this work.

\# Corresponding author

Key words: back pain; genome-wide association study; CHARGE; UK Biobank; pleiotropy. 


\begin{abstract}
Back pain (BP) is a common condition of major social importance and poorly understood pathogenesis. Combining data from the UK Biobank and CHARGE consortium cohorts allowed us to perform a very large GWAS (total $\mathrm{N}=509,070$ ) and examine the genetic correlation and pleiotropy between BP and its clinical and psychosocial risk factors. We identified and replicated three BP associated loci, including one novel region implicating SPOCK2/CHST3 genes. We provide evidence for pleiotropic effects of genetic factors underlying BP, height, and intervertebral disc problems. We also identified independent genetic correlations between BP and depression symptoms, neuroticism, sleep disturbance, overweight, and smoking. A significant enrichment for genes involved in central nervous system and skeletal tissue development was observed. The study of pleiotropy and genetic correlations, supported by the pathway analysis, suggests at least two strong molecular axes of BP genesis, one related to structural/anatomic factors such as intervertebral disk problems and anthropometrics; and another related to the psychological component of pain perception and pain processing. These findings corroborate with the current biopsychosocial model as a paradigm for BP. Overall, the results demonstrate BP to have an extremely complex genetic architecture that overlaps with the genetic predisposition to its biopsychosocial risk factors. The work sheds light on pathways of relevance in the prevention and management of LBP.
\end{abstract}




\section{MAIN}

Back pain (BP) is a common debilitating condition with a lifetime prevalence of $40 \%$ and a very important socioeconomic impact ${ }^{1,2}$. According to the Global Burden of Disease 2016 study, it leads the list of disabling conditions in many parts of the world ${ }^{3}$. Known clinical risk factors for BP include age, female gender and raised body mass index ${ }^{4}$. The greatest risk for episodes of severe BP in population based studies is thought to be attributable to intervertebral lumbar disc degeneration (LDD) ${ }^{5}$, though its predictive and diagnostic impact remains debated ${ }^{6}$. In the majority of episodes of BP the symptoms are transient; however, about $10 \%$ of those experiencing acute BP develop a chronic condition ${ }^{2}$ which places a great socioeconomic burden on $_{\text {society }}{ }^{7-9}$.

There is a clear genetic predisposition to BP with estimates of heritability in the range of 30\%$68 \%{ }^{10-13}$. Similar or higher heritability estimates for LDD have been obtained ${ }^{14,15}$. Importantly, not only is there a phenotypic association between LDD and LBP but a genetic correlation between the two has been reported in twin studies $(11 \%-13 \%)^{10,16}$, suggestive of shared genetic background. Twin studies have demonstrated that BP also shares an underlying genetic predisposition with several of its risk factors including depression and anxiety ${ }^{17}$, educational attainment $^{18}$, obesity ${ }^{19}$ as well as with other pain conditions such as chronic widespread musculoskeletal pain ${ }^{20}$.

We recently performed a genome-wide association study (GWAS) for chronic BP (BP lasting longer than 3 months) from the interim release of the UK Biobank ${ }^{21}$ and from the Cohorts for Heart and Aging Research in Genomic Epidemiology (CHARGE) Musculoskeletal Working Group $^{22}$ (total $\mathrm{N}=158,000$ individuals). Despite a large sample size and relatively well-defined phenotype, the study identified and replicated only three loci associated with chronic BP. This suggests that the genetic architecture of BP is extremely complex and far larger samples are required to make progress in the field.

In the present study we sought to expand the BP GWAS and explore the genetic associations with many of the biopsychosocial risk factors for BP. In brief, we examined 350,000 individuals of European ancestry from the UK Biobank in the discovery phase (91,100 cases and 258,900 controls) followed by a replication phase combining the UK Biobank participants of European, African and Asian ancestry not included in the discovery set, and data from the CHARGE 
cohorts (total $\mathrm{N}=157,752$ ). Post-GWAS analyses included the analysis of pleiotropy, genetic correlations and pathway analyses (Figure 1).

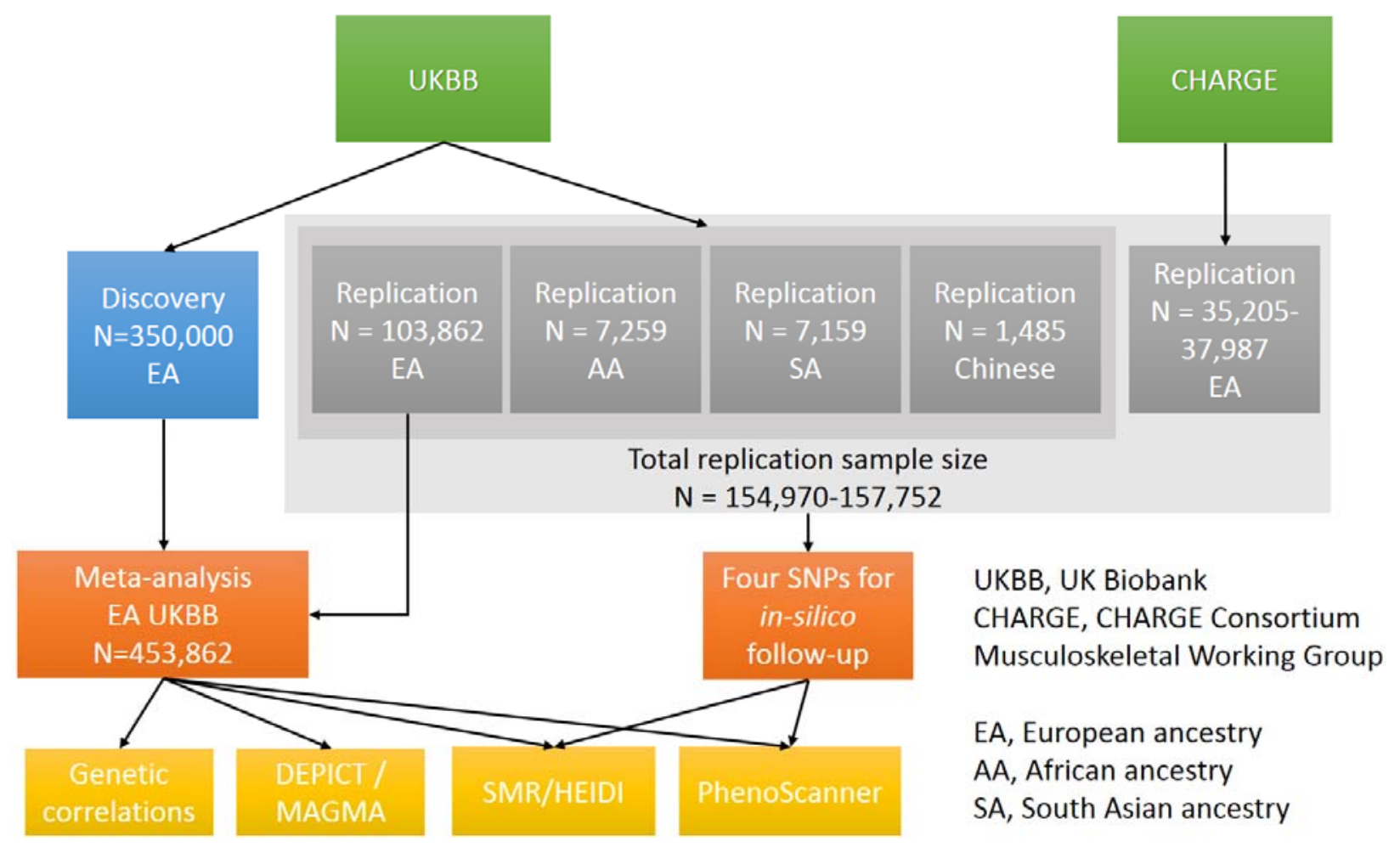

Figure 1. Overview of the study. GWAS for back pain used a combination of UK Biobank and Cohorts for Heart and Aging Research in Genomic Epidemiology (CHARGE) consortium cohorts. Discovery was performed using 350,000 individuals of European ancestry from the UK Biobank. Replication cohorts included individuals of European (EA), African (AA) and South Asian (SA) ancestry and Chinese individuals from the UK Biobank and CHARGE cohorts $(\mathrm{N}=$ 154,970-157,752). Meta-analysis was carried out using the discovery cohort and other individuals of European ancestry from the UK Biobank $(\mathrm{N}=453,862)$ and the results used to estimate genetic correlations with risk factors, establish causal or pleiotropic relationships using summary-data based Mendelian randomization (SMR) followed by heterogeneity in dependent instruments (HEIDI) analysis, and to perform DEPICT and MAGMA analyses to reveal functional relevance. 


\section{RESULTS}

\section{Novel genomic loci associated with back pain}

The discovery sample of white British individuals (as defined by genetic principal components; $\mathrm{N}=350,000$ ) comprised 91,100 BP cases and 258,900 controls, giving a prevalence of BP of $26 \%$. Cases and controls did not differ significantly by age (mean age 57.05 years) or sex (54\% female) (Supplementary Table 1). SNP-based heritability estimated by LD score regression from the BP GWAS was $4 \%$ on the observed scale and $7.3 \%$ on the liability scale. LD-score regression estimated the genomic inflation factor to be 1.29 with intercept of $1.032 \pm 0.009$ (giving the estimate of standardized genomic control inflation factor of $\lambda_{1000}=1.00024$ ), suggesting that most of the inflation was introduced by polygenic effects and that the influence of confounding by population structure and cryptic relatedness was minimal (QQ-plot in Supplementary Figure 1).

After adjusting the results of the discovery GWAS for genomic control factor of 1.032, a total of 183 SNPs positioned over 5 loci remained statistically significant at genome-wide significance level of $\mathrm{p} \leq 5 \times 10^{-8}$ (Figure 2; Table 1). Conditional and joint analysis confirmed that all 5 regions were independent of one another (Supplementary Table 2A). Using meta-analysis for the UK Biobank replication cohorts and the CHARGE Consortium cohorts (total $\mathrm{N}=154,970-157,752$ ), three associations were replicated $(\mathrm{p}<0.01)$ (Supplementary Table 2B): rs12310519 (p = $\left.5.00 \times 10^{-5}\right)$, rs7814941 $\left(\mathrm{p}=5.32 \times 10^{-5}\right)$, and $\mathrm{rs} 3180\left(\mathrm{p}=6.59 \times 10^{-3}\right)$.

Of the three replicated loci, two have been reported previously as associated with other BP phenotypes: the chromosome 12 lead SNP rs12310519 located in the intron of the SOX5 gene was associated with chronic BP in the recent GWAS by the CHARGE and PainOMICS consortia 22. The region on chromosome 8 (lead SNP rs7814941), located in an intergenic site of GSDMC and $C C D C 26$ was identified in a study of sciatica, ${ }^{23}$ and was among the loci associated with chronic BP at $\mathrm{p}<5 \times 10^{-8}$ in the GWAS by the CHARGE and PainOMICS consortia, ${ }^{22}$ but not previously replicated.

The novel replicated locus on chromosome 10 (rs3180 SNP) lies in the region between the 3'UTR of SPOCK2 and downstream of CHST3 genes. This region was previously shown to be 
associated with LDD with the leading SNP rs4148941 reported as a functional variant influencing the CHST3 gene expression level in intervertebral disc tissue ${ }^{24}$. This gene encodes an enzyme which catalyzes sulfation of chondroitin, a component of proteoglycans crucially important in cartilage tissue function and hydration. Rare mutations in CHST3 that disrupt its enzymatic activity have been reported in patients with recessive skeletal abnormalities, including spondyloepiphyseal dysplasia Omani type, Larsen syndrome, humero-spinal dysostosis, and chondrodysplasia with multiple dislocation ${ }^{25-29}$. Another gene in the region, SPOCK2, was previously reported as the positional candidate for bronchopulmonary dysplasia ${ }^{30}$, chromosome 16q carcinogenic deletion (along with CHST3) ${ }^{31}$, and age of smoking initiation ${ }^{32}$. The gene encodes a proteoglycan SPARC/Osteonectin (Cwcv And Kazal Like Domains Proteoglycan 2) involved in extracellular matrix formation and is highly expressed in the central nervous system (CNS) ${ }^{33}$. Using available in-silico instruments we did not find sufficient evidence to determine whether SPOCK2 or CHST3 was the most likely gene associated with BP on chromosome 10 (See Additional results file).

To achieve higher statistical power for the subsequent study of pleiotropic effects and genetic correlations, a meta-analysis of discovery (EA British $\mathrm{N}=350,000$ ) and replication sets (other EA $N=103,862$ ) was performed, giving total $N=453,862$. The SNP-based heritability estimate from this GWAS was $4.2 \%$ on the observed scale and $7.7 \%$ of the liability scale. LD-score regression estimated the genomic inflation factor to be 1.37 with intercept of $1.036 \pm 0.009$ (standardized genomic control inflation factor of $\lambda_{1000}=1.00021$ ). A total of 651 SNPs at 23 loci achieved genome-wide significance threshold of $\mathrm{p}<5 \times 10^{-8}$ (Supplementary Figure 2, Supplementary Tables 3). COJO analysis confirmed that significant loci were all independent of each other. Subsequently, we refer to the results of this meta-analysis as $\mathrm{BP}_{\mathrm{ma}}$ to contrast with the discovery GWAS. 


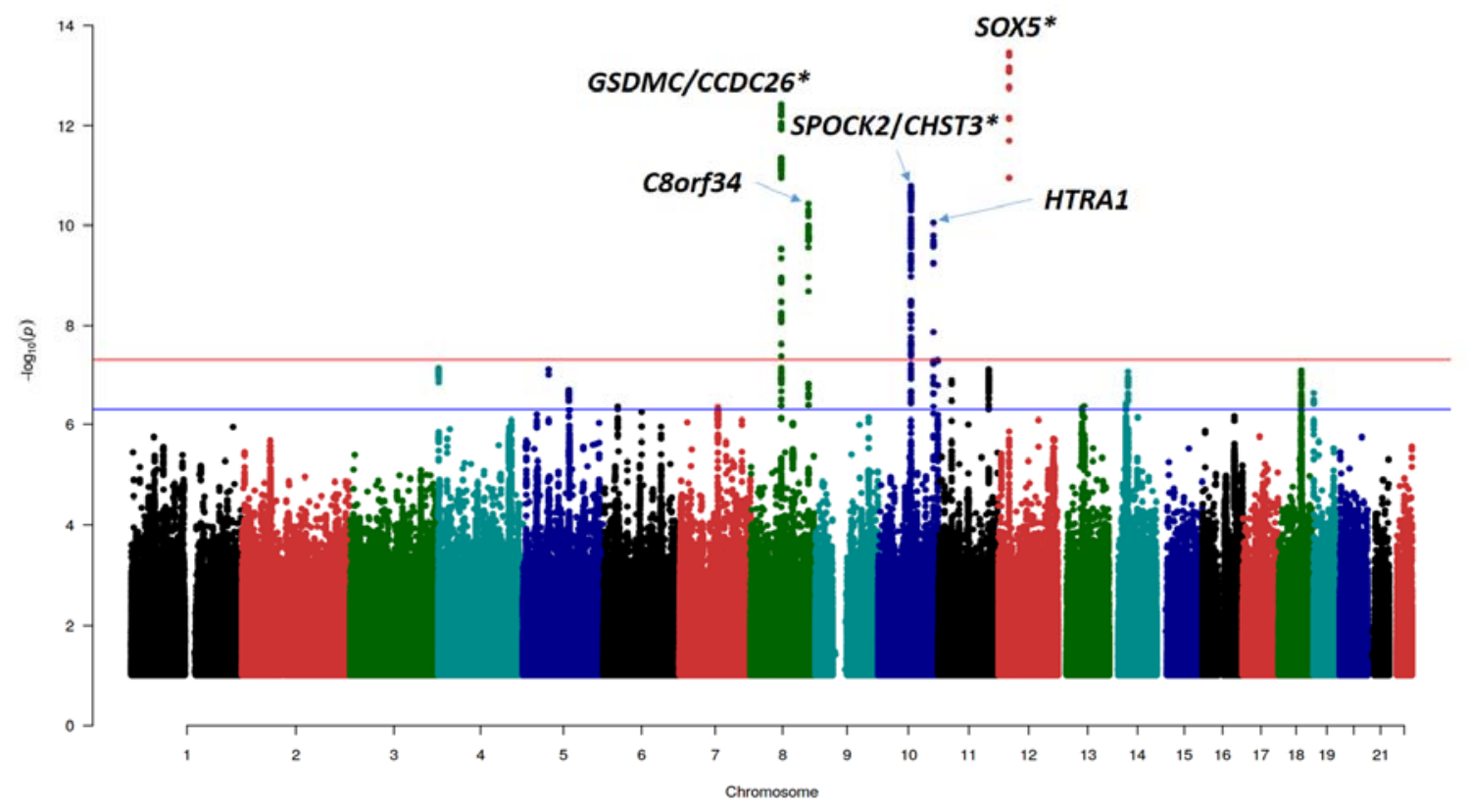

Figure 2. Manhattan plot of discovery GWAS for back pain. Correction was made for genomic control (1.032). The red line corresponds to genome-wide significance threshold of $5 \times 10^{-8}$, while the blue line corresponds to a suggestive association threshold of $5 \times 10^{-7}$. Only SNPs with $\mathrm{p}<0.1$ are presented. Asterisks depict replicated loci.

Table 1. Results of discovery and replication GWAS of BP.

\begin{tabular}{|c|c|c|c|c|c|c|c|c|c|c|}
\hline \multirow[b]{2}{*}{ SNP } & \multirow[b]{2}{*}{ Nearby gene } & \multirow[b]{2}{*}{ Chr:Pos } & \multicolumn{5}{|c|}{ DISCOVERY } & \multicolumn{3}{|c|}{ REPLICATION } \\
\hline & & & $\begin{array}{l}\text { Eff/Ref } \\
\text { Allele }\end{array}$ & EAF & BETA (SE) & P before GC & $P$ after GC & BETA(SE) & $\mathbf{P}$ & $\mathbf{N}$ \\
\hline rs12310519 & SOX5 & 12:23975219 & $\mathrm{C} / \mathrm{T}$ & 0.84 & $-0.056(0.007)$ & $1.38 \times 10^{-14}$ & $3.52 \times 10^{-14}$ & $-0.05(0.011)$ & $5.00 \times 10^{-5}$ & 157618 \\
\hline rs1865442 & C8orf34 & $8: 69574165$ & $\mathrm{C} / \mathrm{T}$ & 0.82 & $-0.052(0.007)$ & $1.60 \times 10^{-13}$ & $3.79 \times 10^{-13}$ & $-0.03(0.011)$ & $1.05 \times 10^{-2}$ & 157724 \\
\hline rs3180 & SPOCK2/CHST3 & 10:73820622 & $\mathbf{A} / \mathbf{G}$ & 0.44 & $-0.037(0.006)$ & $7.83 \times 10^{-12}$ & $1.65 \times 10^{-11}$ & $-0.02(0.008)$ & $6.59 \times 10^{-3}$ & 157731 \\
\hline rs7814941 & GSDMC/CCDC26 & 8:130718859 & $\mathbf{A} / \mathbf{G}$ & 0.80 & $0.046(0.007)$ & $1.81 \times 10^{-11}$ & $3.71 \times 10^{-11}$ & $0.04(0.010)$ & $5.32 \times 10^{-5}$ & 157728 \\
\hline rs2672596 & HTRAl & $10: 124226793$ & G/A & 0.74 & $0.041(0.006)$ & $4.46 \times 10^{-11}$ & $8.89 \times 10^{-11}$ & $0.02(0.010)$ & $3.98 \times 10^{-2}$ & 157735 \\
\hline rs10870267 & DPYSLA & $10: 133968063$ & $\mathrm{C} / \mathrm{T}$ & 0.44 & $0.030(0.006)$ & $3.09 \times 10^{-8}$ & $5.05 \times 10^{-8}$ & - & - & - \\
\hline rs4974563 & SPON2 & $4: 1138153$ & $\mathrm{C} / \mathrm{T}$ & 0.30 & $-0.032(0.006)$ & $4.39 \times 10^{-8}$ & $7.10 \times 10^{-8}$ & - & - & - \\
\hline rs2910576 & $P L K 2$ & $5: 57532748$ & $\mathrm{~A} / \mathrm{T}$ & 0.19 & $0.038(0.007)$ & $4.71 \times 10^{-8}$ & $7.60 \times 10^{-8}$ & - & - & - \\
\hline rs7105462 & NCAM1 & 11:112912048 & G/A & 0.41 & $0.030(0.006)$ & $4.72 \times 10^{-8}$ & $7.62 \times 10^{-8}$ & - & - & - \\
\hline rs10502966 & $D C C$ & $18: 50748499$ & $\mathrm{~A} / \mathrm{G}$ & 0.58 & $-0.030(0.006)$ & $4.94 \times 10^{-8}$ & $7.96 \times 10^{-8}$ & - & - & - \\
\hline
\end{tabular}

Table legend: Chr:Pos - physical position of the SNP; Eff/Ref Allele - Effect and Reference alleles; EAF - effect allele frequency; BETA (SE) - effect of the SNP and standard error; P before GC - p-value before genomic control; $\mathrm{P}$ after GC - p-value after genomic control; $\mathrm{N}$ - sample size of replication. Bold font indicates the SNPs that passed the thresholds for statistical significance $\left(5 \times 10^{-8}\right.$ for the discovery and 0.01 for replication phases, respectively). 


\section{Causal and pleiotropic effects of genetic factors underlying back pain and its risk factors}

\section{Identifying causal genes via a study of gene expression}

For replicated regions we aimed to identify genes whose expression might mediate the association between SNP and BP. We performed a summary-data based Mendelian randomization (SMR) analysis followed by heterogeneity in dependent instruments (HEIDI) analysis ${ }^{34}$ using eQTL data from a range of tissues including blood ${ }^{35}$ and 44 tissues provided in the GTEx v. 6p database ${ }^{36}$ (Supplementary table 4A). In short, SMR tests the association between gene expression in a particular tissue and a trait using the most highly associated SNP as a genetic instrument. A significant SMR test indicates that a given functional variant determines both gene expression and the trait of interest via causality or pleiotropy, but it may also suggest that functional variants underlying gene expression are in linkage disequilibrium with those controlling the trait. Inference of whether a functional variant mediates both BP and gene expression were made based on the HEIDI test: $\mathrm{P}_{\text {HEIDI }} \geq 0.01$ (likely shared causal SNP) and $\mathrm{P}_{\text {HEIDI }}<0.01$ (sharing of a causal SNP is unlikely). Results are presented in Supplementary Table 4B.

We observed a statistically significant SMR $\left(\mathrm{p}<3 \times 10^{-5}\right)$ and no difference in association patterns for the rs3180 locus and SPOCK2 in blood $\left(\beta_{\mathrm{SMR}}=5.9 ; \mathrm{p}_{\mathrm{SMR}}=1.0 \times 10^{-8}\right)$ and in adrenal gland $\left(\beta_{\mathrm{SMR}}=-20.6 ; \mathrm{p}_{\mathrm{SMR}}=1.3 \times 10^{-6}\right)$. Moreover, for this locus we detected three probes with suggestive significance SMR coefficient and $\mathrm{p}_{\text {HEIDI }}>0.01$ : two probes for CHST3 gene in testis $\left(\beta_{\mathrm{SMR}}=6.3 ; \mathrm{p}_{\mathrm{SMR}}=5.5 \times 10^{-5}\right)$ and in EBV-transformed lymphocytes $\left(\beta_{\mathrm{SMR}}=-17.1 ; \mathrm{p}_{\mathrm{SMR}}=\right.$ $\left.1.7 \times 10^{-4}\right)$; and one probe for SPOCK2 gene in muscle skeletal tissue $\left(\beta_{\mathrm{SMR}}=-9.6 ; \mathrm{p}_{\mathrm{SMR}}=8.7 \times 10^{-}\right.$ ${ }^{5}$ ). The results suggest that either SPOCK2 or CHST3 or both are possible causal genes for BP in the region tagged by rs3180. It is worth noting, though, that some of the tissues with significant findings in this analysis (testis, EBV-transformed lymphocytes, adrenal gland) do not seem relevant to BP in an anatomical or functional sense. Nevertheless, a BP-associated variation in SPOCK2/CHST3 region was linked with CHST3 expression in intervertebral disc tissue in an in vitro functional study previously ${ }^{24}$.

For the locus tagged by rs7814941, we detected two expression probes with statistically significant SMR coefficients that corresponded to GSDMC gene. However, in all cases there was a significant $\left(\mathrm{p}_{\text {HEIDI }}<2 \times 10^{-7}\right.$ ) difference in association patterns between the SNP and gene 
expression and BP. This suggests that the association between this region and BP is unlikely driven by variation in GSDMC gene expression.

\section{Pleiotropic effects of genetic variants associated with BP and other complex traits}

Using the SMR/HEIDI approach, we also tested for potential pleiotropy of effects of three BP loci on seventeen known risk factors or related conditions for which data were available in public databases: osteoarthritis, self-reported intervertebral disc problems, osteoporosis, scoliosis, smoking status, standing height, BMI, well-being (happiness), fluid intelligence score, educational attainment (years of education), anxiety/panic attacks, depression and the 'Big Five' personality traits (openness to experience, conscientiousness, extraversion, agreeableness, and neuroticism) (Supplementary Table 4C; Supplementary methods).

Results are presented in Supplementary Table 4D. Statistically significant $\left(\mathrm{p}<9.8 \times 10^{-4}\right)$ SMR coefficients were revealed for height with variants rs7814941 and rs3180 ( $\mathrm{p}_{\mathrm{SMR}}=3.60 \times 10^{-13}$ and $4.35 \times 10^{-5}$, respectively). Locus rs7814941 showed significant heterogeneity in association patterns with height $\left(\mathrm{p}_{\text {HEIDI }}=5.58 \times 10^{-12}\right.$ ) suggesting the presence of different functional variants for height and BP at this locus. Locus rs3180 showed no heterogeneity in association patterns between BP and height in HEIDI ( $\mathrm{p}_{\text {HEIDI }}=0.79$ ), thus suggesting pleiotropy - the same functional variant(s) was influencing both traits. All three loci demonstrated significant SMR results with intervertebral disc problems $\left(\mathrm{p}_{\mathrm{SMR}}=3.30 \times 10^{-7}, 3.75 \times 10^{-7}\right.$, and $3.17 \times 10^{-5}$, for rs 3180 , rs 12310519 , and rs7814941, respectively; Table 2); with all three showing no heterogeneity in association patterns between BP and intervertebral disc problems (all $\mathrm{p}_{\mathrm{HEIDI}}>0.01$ ); and in all cases SMR coefficient was positive suggesting that the same causal genetic factors attributable to these loci increase the risk of both BP and self-reported intervertebral disc problems. 
Table 2. Results of summary-level Mendelian randomization and pleiotropy analysis of SNPs associated with BP

\begin{tabular}{|c|c|c|c|c|}
\hline \multirow[t]{3}{*}{ Trait } & \multirow[t]{3}{*}{ Statistics } & \multicolumn{3}{|c|}{ SNP (Gene) } \\
\hline & & rs3180 & rs12310519 & rs7814941 \\
\hline & & (SPOCK2/CHST3) & $(\mathrm{SOX5})$ & $(G S D M C / C C D C 26)$ \\
\hline \multirow[t]{3}{*}{ Height } & $\beta_{\mathrm{SMR}}$ & -1.677 & 0.800 & 6.013 \\
\hline & $\mathrm{p}_{\mathrm{SMR}}$ & $4.3 \times 10^{-5}$ & $7.4 \times 10^{-3}$ & $3.6 \times 10^{-13}$ \\
\hline & $\mathrm{p}_{\text {HEIDI }}$ & 0.79 & - & $5.6 \times 10^{-12}$ \\
\hline \multirow{3}{*}{$\begin{array}{l}\text { Intervertebral } \\
\text { disc problems }\end{array}$} & $\beta_{\mathrm{SMR}}$ & 0.068 & 0.050 & 0.040 \\
\hline & $\mathrm{p}_{\mathrm{SMR}}$ & $3.3 \times 10^{-7}$ & $3.8 \times 10^{-7}$ & $3.2 \times 10^{-5}$ \\
\hline & $\mathrm{p}_{\text {HEIDI }}$ & 0.75 & 0.12 & 0.50 \\
\hline
\end{tabular}

Table legend: Results of SMR/HEIDI tests using data from GeneAtlas. For the HEIDI tests, a hypothesis of pleiotropy was rejected at $\mathrm{p}<0.01$; with $\mathrm{p}>0.01$, we considered pleiotropy as a likely explanation. Two traits (height and intervertebral disc problems) with at least one significant SMR coefficient $\left(\mathrm{p}<9.8 \times 10^{-4}\right)$ among three loci are presented. $\beta_{\text {SMR }}$ is SMR coefficient; $p_{S M R}$ is $p$-value for SMR test; $p_{\text {HEIDI }}$ is $p$-value for HEIDI test (not calculated if $\mathrm{p}_{\mathrm{SMR}}$ was insignificant, “-“).

\section{Back pain shares genetic components with psychiatric, sociodemographic and anthropometric traits}

To establish shared genetic components between BP and other complex traits, we carried out an agnostic analysis of 225 complex traits available in LD-hub. We observed a significant genetic correlation $\left(\mathrm{p}<4.4 \times 10^{-5}\right)$ between $\mathrm{BP}_{\mathrm{ma}}$ and 33 traits (Supplementary Table 6, Supplementary Figure 3$)$, with the strongest positive correlations $\left(\rho_{\mathrm{g}}>0.35\right)$ found with BP and neuroticism ${ }^{37}$ $\left(\rho_{\mathrm{g}}=0.49\right)$, insomnia ${ }^{38}\left(\rho_{\mathrm{g}}=0.46\right)$, depressive symptoms ${ }^{37}\left(\rho_{\mathrm{g}}=0.53\right)$ and major depressive disorder ${ }^{39}\left(\rho_{\mathrm{g}}=0.39\right)$. The strongest negative correlations $\left(\rho_{\mathrm{g}}<-0.35\right)$ were between $\mathrm{BP}_{\mathrm{ma}}$ and age of first birth ${ }^{40}\left(\rho_{\mathrm{g}}=-0.49\right)$, years of schooling ${ }^{41}\left(\rho_{\mathrm{g}}=-0.47\right)$, mothers age at death ${ }^{42}\left(\rho_{\mathrm{g}}=-0.43\right)$, parents age at death ${ }^{42}\left(\rho_{\mathrm{g}}=-0.38\right)$ and college completion ${ }^{43}\left(\rho_{\mathrm{g}}=-0.51\right)$. The traits exhibiting strong genetic correlation with BP fell into several distinct clusters (Figure 3): 1) the cluster of obesity-related traits, 2) the cluster related to mood and sleep, and 3) the cluster related to sociodemographic factors (including education) and smoking. 
To identify which pair-wise genetic correlations were conditionally independent of each other, we calculated partial genetic correlations for $\mathrm{BP}_{\mathrm{ma}}$ and 8 traits selected from each subcluster (using a distance threshold of 0.5 on a hierarchical clustering dendrogram) of the genetic correlation matrix (Figure 4, Supplementary Figure 4). In short, partial correlation is the measure of association between two variables while controlling for the effect of one or more additional variables. This analysis found such traits as "mother age of death", "lung cancer", and "former vs current smoking", and "age of first birth" to not be independently correlated with BP. Partial correlations for depressive symptoms and sleep duration were similar to the pair-wise correlations. Finally, partial correlations with BP for "waist circumference" and "college completion" were much smaller than the pair-wise correlations but remained statistically significant $\left(\mathrm{p}<4.4 \times 10^{-5}\right)$.

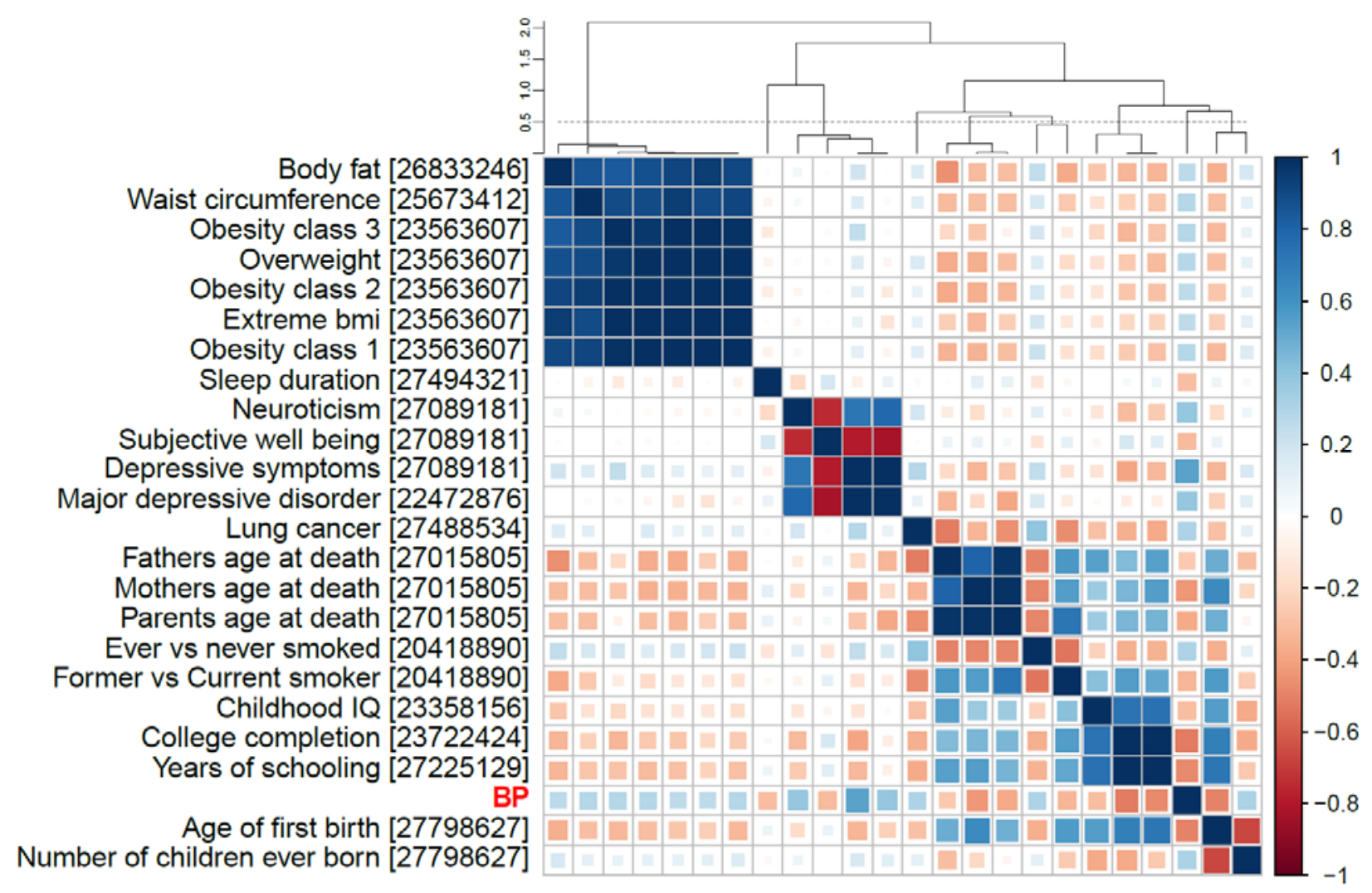

Figure 3. Heatmap for 23 traits with strongest statistically significant genetic correlations with back pain (absolute $\rho_{\mathrm{g}} \geq \mathbf{0 . 2 5} ; \mathbf{p} \leq \mathbf{4 . 4 \times 1 0 ^ { - 5 }}$ ). Hierarchical clustering was carried out based on genetic correlations between all pairs of traits. PMID references are placed in square brackets. The dashed line on the cluster dendrogram refers to the threshold of 0.5 , depicting 9 subclusters (including BP). 


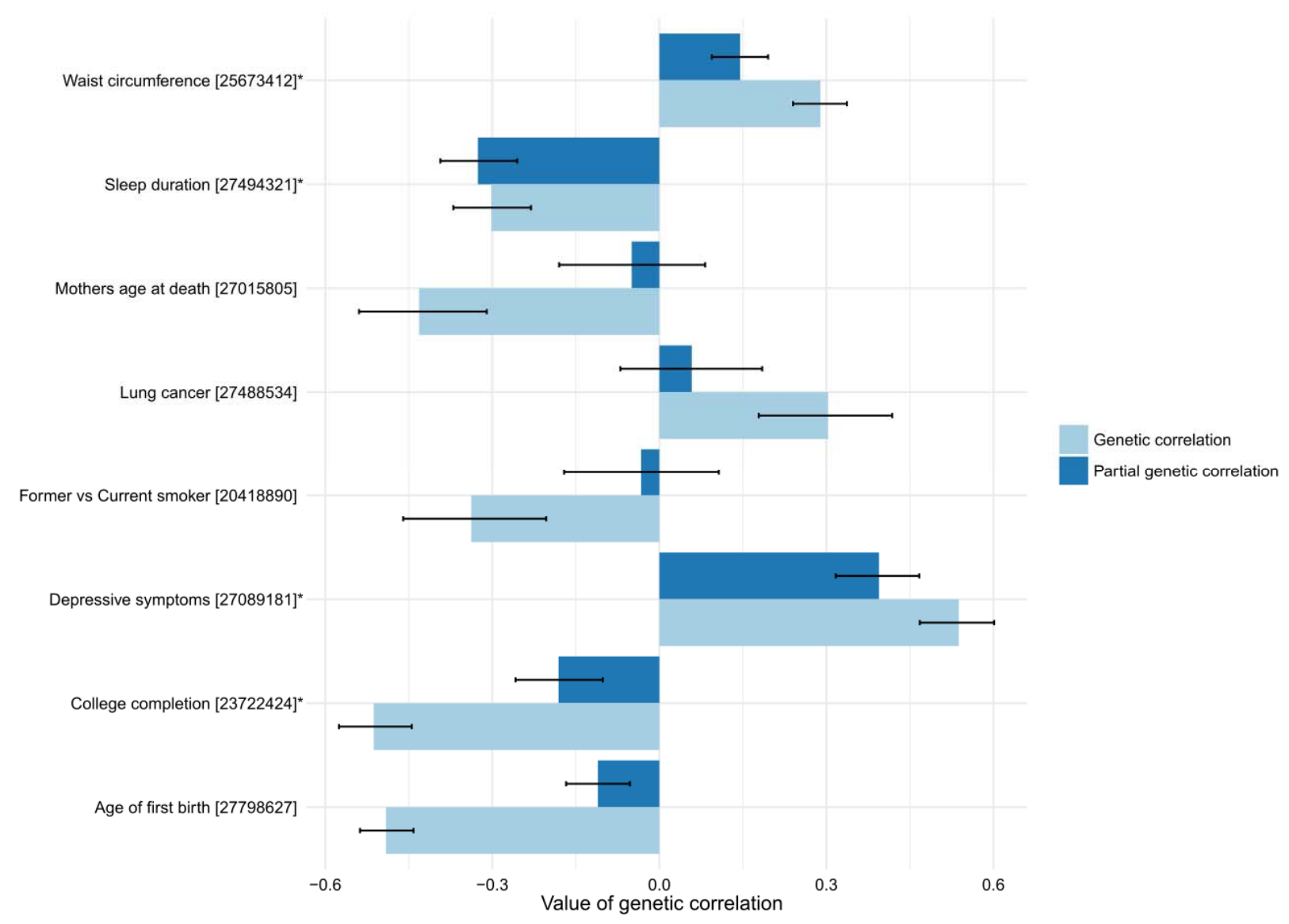

Figure 4. Partial genetic correlation and pair-wise genetic correlation barplots for 8 traits (one trait from each subcluster with threshold of 0.5 on hierarchical clustering dendrogram of genetic correlation matrix). Error bars correspond to 95\% confidence intervals. Asterisks depict traits in which partial correlation with BP is significant $\left(\mathrm{p}<4.4 \times 10^{-5}\right)$.

In addition to an agnostic analysis of all complex traits from LD-hub, we also carried out a focused analysis of genetic correlations between BP and 17 complex traits considered as risk factors for BP: self-reported osteoarthritis, self-reported intervertebral disc problems, selfreported osteoporosis, scoliosis, smoking status, standing height, BMI, happiness, fluid intelligence score, years of education, anxiety/panic attacks, depression and Big Five traits (Supplementary Table 4C). The strongest positive correlations were found for self-reported intervertebral disc problems $\left(\rho_{\mathrm{g}}=0.77, \mathrm{p}=6.7 \times 10^{-24}\right)$; self-reported osteoarthritis $\left(\rho_{\mathrm{g}}=0.55, \mathrm{p}=\right.$ $\left.7.5 \times 10^{-41}\right)$; and depression $\left(\rho_{\mathrm{g}}=0.44, \mathrm{p}=1.3 \times 10^{-23}\right)$. The strongest negative correlation was found for education attainment $\left(\rho_{\mathrm{g}}=-0.47, \mathrm{p}=7.1 \times 10^{-101}\right)$. Scoliosis, smoking status and BMI 
had moderate positive genetic correlation with $\mathrm{BP}_{\mathrm{ma}}\left(\rho_{\mathrm{g}}=0.35,0.35\right.$ and 0.33 respectively, with $\mathrm{p}=0.001,7.3 \times 10^{-42}$ and $2.0 \times 10^{-56}$ respectively). Overall, the results of the analysis of the risk factors were consistent with the analysis of 225 traits.

\section{Genetic factors underlying back pain are involved in neurological pathways}

We used DEPICT with all independent variants (as identified by COJO analysis) from $\mathrm{BP}_{\text {ma }}$ with $\mathrm{p}<1 \mathrm{e}-5$ (227 SNPs in total; Supplementary Table 6A-C) and identified potential enrichment of gene sets $(\mathrm{FDR}<0.2)$ related to nervous system development and skeletal muscle development. We did not identify a significant enrichment of expression across any tissues and cell types (FDR > 0.2), although we observed a trend towards enrichment of components of CNS (Supplementary Table 7A-C). Similar results were observed when analyzing enrichment of expression of genes located around $23 \mathrm{BP}_{\mathrm{ma}}$ independent genome-wide significant variants (Supplementary Table 7D-F).

Analysis by MAGMA ${ }^{44}$ revealed three significant gene sets (Supplementary Table 8): M12307 ("Nikolsky breast cancer 16q24 amplicon", FDR=0.02; copy number amplicons of 53 genes enriched with major tumorigenic pathways and breast cancer-causative genes ${ }^{45}$ ), GO:0051590 (positive regulation of neurotransmitter transport, FDR=0.02) and GO:0021952 (central nervous system projection neuron axonogenesis, FDR=0.02). Tissue expression analysis for 30 general tissue types revealed significant enrichment of expression in brain (FDR=0.01). 


\section{DISCUSSION}

The current study is the largest genetic association study to date for BP and included more than 500,000 individuals. The results provide insights into the genetic composition of predisposition to BP, one of the leading causes of disability worldwide. We quadrupled the number of genomewide significantly associated BP loci (from five ${ }^{22}$ to 23 ), and increased the number of replicated BP loci from one to three. Our work has implicated two new positional candidate genes: SPOCK2 and CHST3. The region where these genes reside has previously been described as associated with LDD in Chinese individuals, and in vitro functional study suggested a mechanism linking variation in this locus (specifically, rs4148941) and expression of CHST3, a functionally highly plausible gene ${ }^{24}$. Our in silico functional analysis, however, suggests that the closely adjacent SPOCK2 gene may be another candidate in the region. In particular, we provide evidence of relationships between both SPOCK2 and CHST3 gene expression and the risk of BP. At the same time, using available in-silico instruments, we couldn't provide enough evidence in favor of SPOCK2 or CHST3 as the most likely gene associated with BP on the locus on chromosome 10 (see Additional results file).

We found evidence of pleiotropic effects for the genetic factors underlying BP, height, and intervertebral disc problems. From epidemiological studies, both height and LDD are known to be associated with $\mathrm{BP}$ and have been proposed to have causal effects on $\mathrm{BP}{ }^{5,46}$. The genetic pleiotropy identified in the current study provides insight into the molecular background underlying these associations. Importantly, while only one of the three loci (rs3180) exhibited pleiotropic effects for BP and height, all three loci demonstrated pleiotropy for BP and selfreported intervertebral disk problems. In addition, the observed genetic correlation between height and BP was small and statistically insignificant $\left(\rho_{\mathrm{g}}=0.05, \mathrm{p}=0.07\right)$, while the genetic correlation between intervertebral disc problems and BP was high and statistically significant $\left(\rho_{g}\right.$ $\left.=0.77, \mathrm{p}=6.7 \times 10^{-24}\right)$. These results suggest stronger shared underlying genetic factors between intervertebral disk degeneration and BP, as compared to height and BP, in keeping with the epidemiological evidence of a stronger association of BP with disc degeneration ${ }^{16,47}$ and a weaker association with height. ${ }^{48}$ An alternative explanation for our observation that loci influencing BP also affect intervertebral disc problems might be an overlap between individuals reporting BP and intervertebral disc problems in UK Biobank. However, at least for the lead 
SNPs tagging regions near SOX5 (rs12310519), and GSDMC/CCDC26 (rs7814941 via proxy rs4733724), nominally significant associations $\left(\mathrm{p}=1.1 \times 10^{-4}\right.$ and $\mathrm{p}=0.023$, respectively) with MRI-proven LDD were found in a meta-analysis of 4600 individuals independent from the current study sample and not selected by BP status. ${ }^{49}$ This strengthens the argument in support of a shared genetic basis for BP and intervertebral disc problems.

To our knowledge, this is the first study to use contemporary quantitative genetic methods in an attempt to replicate the results of twin studies examining shared genetic influences on BP with other traits, including putative BP risk factors. ${ }^{10,16,47,50-54}$ In so doing, we took the broadest approach to date and examined a wide range of complex traits and known risk factors, revealing three clusters sharing significant genetic correlations with BP : the obesity-related traits, the mood and sleep related traits, and the sociodemographic factors (including education) and smoking. Moreover, we identified mutually independent genetic correlations between BP and depression, sleep disturbance, waist circumference and college completion. The magnitude and direction of many of the observed genetic correlations in the current study follow from the results of classic epidemiology and genetic epidemiology studies of BP suggesting, perhaps, that the environmental components to these risk factors have been overstated or at least themselves have a genetic basis. For instance, we observed strong positive genetic correlations between BP and depression related phenotypes, and between BP and obesity-related traits. These traits are known to often co-occur with BP and twin studies have suggested that they share underlying genetic factors ${ }^{17}$, with similar genetic correlations also seen for other pain phenotypes. ${ }^{55-57}$ Our results confirm a recent twin report of genetic correlation of sleep disturbance with $\mathrm{BP}{ }^{58}$ Overall, the analysis of genetic correlations provides evidence for shared molecular pathways underlying BP and traits considered as BP risk factors, thus providing basis for identification of causal links between them.

Our pathway analysis revealed the importance of genetic factors in CNS and skeletal muscle in BP. While the CNS has long been recognized as the key component in the pathogenesis of chronic pain, ${ }^{59}$ the role of skeletal muscle is still not well defined. ${ }^{60,61}$ Altogether, these data provide a starting point for further functional analyses of mechanisms underlying BP (Figure 5). The study of pleiotropy and genetic correlations, supported by the pathway analysis, suggests at least two strong molecular axes of BP genesis, one related to structural/anatomic factors such as intervertebral disk problems and anthropometrics; and another related to the psychological 
component of pain perception and pain processing. These two axes correspond roughly to the different "biomedical" and "biopsychosocial" viewpoints that have dominated BP research and clinical care for the past several decades. ${ }^{62}$ Pathway analysis also produced an unexpected enrichment for genes involved in "Nikolsky breast cancer 16q24 amplicon" gene set. This gene set includes 53 genes and represents one of 30 genomic regions with copy number gain found in the analysis of 191 breast tumours ${ }^{45}$. It is not known to be enriched for pain-related or other relevant pathways; therefore, its relationship with BP needs to be explored further.

Despite the study of close to half a million people, we identified and replicated only 3 loci. Together with the evidence of relatively high heritability from twin studies ${ }^{10-13}$, this suggests that BP is genetically a very complex, highly polygenic phenotype. In part, this can be explained by the heterogeneity of the phenotype itself, as BP likely occurs for a vast range of reasons, many having different underlying molecular pathology ${ }^{63}$. Our approach used a standard definition of "any back pain" in the discovery stage, but permitted some heterogeneity with respect to $\mathrm{BP}$ duration among cohorts included in the replication stage (any BP in the UK Biobank sub-cohorts vs chronic BP in the CHARGE cohorts). Future progress of genetic studies of BP would benefit from more consistent phenotyping. On the other hand, our experience to date of working with back pain consortia - with no more than 3 cohorts having closely comparable back pain definitions and question items - has shown that it is extremely difficult to bring together cohorts of comparable size having uniform phenotype definition. This reflects the current state of BP research, where there is no universally accepted gold standard for defining $\mathrm{BP}^{22}$ Recently established consensus guidelines for core BP definitions may facilitate future efforts to harmonize definitions between cohorts. ${ }^{64}$ 


\section{Candidate genes}

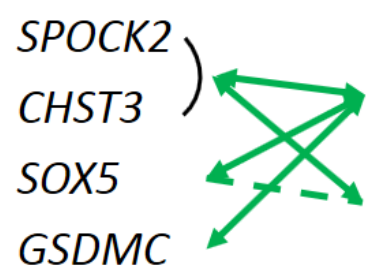

\section{Genetic correlations}

- Obesity-related traits

- Traits related to mood and sleep

- Traits related to sociodemographic factors

(including education and smoking)

\section{Pleiotropy}

(SMR-HEIDI)

Intervertebral disc

problems

Height

\section{Pathways \\ (DEPICT, MAGMA)}

- Potential enrichment for gene sets (FDR $<0.2$ ) related to regulation of nervous system development and skeletal muscle development

- GO:0051590, positive regulation of neurotransmitter transport (FDR=0.02)

- GO:0021952, central nervous system projection neuron axonogenesis ( $F D R=0.02$ )

\section{The formation of the spine / cartilage / bone / connective tissues}

\section{Signaling / neurogenesis}

Figure 5. Summary of genes and pathways in back pain. Left part of the figure summarizes information about positional candidate genes and genetic correlations. Green arrows depict pleiotropy by SMR/HEIDI method. Dashed green lines depict suggested pleiotropy by SMR/HEIDI. Left part of the figure summarizes the results of pathway analyses. 


\section{ONLINE METHODS}

\section{Phenotype definition}

The study was based on data from the UK Biobank Resource ${ }^{21}$ and cohorts from CHARGE Consortium Musculoskeletal Working Group. For UK Biobank cases of BP were defined as those who reported "Back pain" in the response to the question: "Pain type(s) experienced in last month". Controls were defined as those who did not report BP in response to this question. Individuals who did not reply or replied: "Prefer not to answer" or "Pain all over the body" were excluded.

For CHARGE Consortium cases were defined as those reporting BP present for at least 3 months, while the controls were defined as those who reported no BP or BP with shorter duration. ${ }^{65}$ Thus, the definition of BP in these cohorts corresponded to chronic BP.

\section{Sample}

The available sample from UK Biobank included 487,409 individuals with imputed data. For the discovery set we selected at random 350,000 British individuals of European ancestry (EA) according to the genetic principal components provided by the UK Biobank (Supplementary Table 1).

For replication, we used a combination of the UK Biobank participants not included in the discovery set, and from the CHARGE Consortium ${ }^{22}$. Replication cohorts from the UK Biobank comprised rest of EA individuals $(n=103,862)$, individuals of African ancestry $(A A, n=7,259)$, individuals of South Asian ancestry (Indian, Pakistani, and Bangladeshi; n = 7,159), and Chinese individuals $(\mathrm{n}=1,485)$. The CHARGE Consortium provided data for EA individuals from 15 cohorts (total $n=35,205-37,987$ ). To reduce the risk of bias due to population stratification, all these groups were analysed separately followed by a meta-analysis. Total resulting sample size for replication was 154,970-157,752 individuals (Supplementary Table 1).

\section{Statistical analysis}

\section{Genome-wide association testing}

PLINK 2.0 was used to carry out the genome-wide association analysis in the UK Biobank discovery and replication samples. Imputed genotype provided by the UK Biobank were used ${ }^{21}$ and only SNPs from the list of HRC imputed SNPs were analysed (http://www.haplotype- 
reference-consortium.org/site) due to reported issue with SNPs imputed using 1000 Genomes panel (http://www.ukbiobank.ac.uk/2017/07/important-note-about-imputed-genetics-data/). Logistic regression was used to evaluate additive genetic effects of the single nucleotide polymorphisms (SNPs) for BP as a binary trait adjusting for age, sex, genotyping array type, and 10 genetic principal components provided by UK Biobank.

The following filters were applied: minor allele count $\geq 100$; deviation from Hardy-Weinberg equilibrium p-value $\geq 1 \mathrm{e}-6$; genotyping call rate $\geq 0.98 \%$; individual call rate $\geq 0.98 \%$; and imputation quality score $\geq 0.7$ (MACH r2 calculated by PLINK 2.0). Only biallelic markers were used and SNPs that had the same rsID in different genomic locations were excluded.

\section{Conditional and joint multi-SNP analysis}

Conditional and joint analysis (COJO) as implemented in the program GCTA ${ }^{66}$ was used to find SNPs independently associated with the phenotype. As the input, this method uses summary statistics and a reference sample that is utilised for the LD estimation. We performed the analyses using $\mathrm{p}=5 \times 10^{-8}$ and $\mathrm{p}=1 \times 10^{-5}$ as the genome-wide significance and suggestive significance thresholds respectively. For the LD reference, we used a sample of 10,000 British EA individuals randomly selected from 350,000 people used in the GWAS discovery phase.

\section{Replication and meta-analysis}

Replication was performed by meta-analysis of all replication cohorts for loci selected at the discovery phase. Replication significance threshold was set as p-value $<0.01$ (Bonferroni corrected 0.05/5). Subsequent analyses of heritability, genetic correlation, and functional investigation used the results of meta-analysis of the discovery cohort and replication cohort of EA individuals from UK Biobank $(\mathrm{N}=453,862)$. METAL software ${ }^{67}$ was applied using inversevariance-weighted approach.

LD hub ${ }^{68}$ and ldsc ${ }^{69}$ tools were used to estimate SNP-captured heritability. Summary statistics files were filtered using ldsc software with default options (r2>0.9, MAF>0.01 and the overlap with "high quality SNPs" - a total of 1,215,001 common HapMap3 SNPs with high imputation quality). The HLA region on chromosome 6 was excluded. These SNPs were used for the further analysis of heritability and genetic correlations as well as to estimate genomic control inflation factor lambda (intercept) ${ }^{70}$. 


\section{Genetic correlation analyses}

Genetic correlations were estimated using the BP meta-analysis results $(\mathrm{N}=453,862)$, not including the CHARGE cohorts. Two sets of traits were analyzed. First included a total of 225 traits available on LD-hub after removing duplicates via using only the most recent study for each trait as indicated by the largest PMID number. Another set comprised traits considered by us as risk factors for BP: self-reported osteoarthritis, self-reported intervertebral disc problems, self-reported osteoporosis, scoliosis, smoking status, standing height, BMI, happiness, fluid intelligence score, years of education, anxiety/panic attacks, depression and Big Five personality traits (openness to experience, conscientiousness, extraversion, agreeableness, and neuroticism). Genetic correlations between BP and 225 traits were considered statistically significant at pvalue $\leq 4.4 \times 10^{-5}$ (Bonferroni corrected, 0.01/225). To visualize the results, we focused on genetic correlations of greatest magnitude and selected only the traits with absolute values of genetic correlation with $\mathrm{BP}>0.25$. This filtering led to a total of 23 traits (excluding BP). Clustering and visualization was carried out using "corrplot" package for R and basic "hclust" function. For clustering, we estimated squared Euclidean distances by subtracting absolute values of genetic correlation from 1 and used Ward's clustering method.

To obtain genetic correlations that were independent from each other, we estimated partial genetic correlations for a subset of traits using the inverse of correlation matrix (free of collinearity) followed by the correlation estimate given by the equation $\rho_{i j}=-\frac{p_{i j}}{\sqrt{p_{i i} * p_{j j}}}$. We selected for this analysis one trait from each subcluster (using distance threshold of 0.5 on hierarchical clustering dendrogram) of the matrix of genetic correlations for 24 traits (we selected only the trait with the highest absolute value of its correlation with BP). This selection led to 8 traits in total.

\section{In silico functional analysis}

\section{VEP and credible set}

Functional annotation of SNPs was carried out using variant effect predictor (VEP) software ${ }^{71}$ with GRCH37 genomic reference. For each studied locus we selected the set of SNPs (credible set) that had strong associations and the most probably had causal influence on BP. We used PAINTOR software ${ }^{72}$ to prepare the credible set of SNPs. For this analysis, we provided 
PAINTOR with clumping results, LD matrices and annotation files. Using PLINK1.9 and 10,000 samples reference set described above (the same subset as used in COJO and DEPICT analyses) we performed clumping analysis with 'p1' and 'p2' p-value threshold parameters set to $5 \times 10^{-8}$, 'r2' set to 0.1 and MAF>0.002. Then, using the same reference set we generated pair-wise correlation matrix for all SNPs in each region in clumping analysis results using PLINK "--r" option. When running PAINTOR, we did not use annotations; we changed options controlling input and output files format only, and otherwise we have used default parameters. In the next step, all output results were aggregated into one file and SNPs marked by PAINTOR as $99 \%$ credible set were chosen for further functional annotation.

\section{SMR/HEIDI analysis}

Potential pleiotropic effects of genetic variants on BP and other traits were tested using summary data-based Mendelian randomization (SMR) analysis and heterogeneity in dependent instruments (HEIDI) method ${ }^{34}$. SMR-HEIDI is analogous to conventional Mendelian randomization and may be conducted using summary level GWAS data. In short, the first method tests for association between the traits of interest mediated by a locus, and the second test identifies whether the traits are affected by the same underlying causal variant. This analysis was carried out for SNPs associated with BP in the current study. Briefly, starting with an index SNP, we screened for traits which may be affected by genetic variation in the same region, and then performed a pleiotropy vs linkage disequilibrium test. In the screening stage, we used a limited list of traits including 19 traits considered risk factors for BP (19 traits in total; Supplementary table 4C). To perform HEIDI analysis, regional summary level GWAS results are required, including regression coefficients and respective standard errors. Such data were available for seventeen traits: self-reported osteoarthritis, self-reported intervertebral disc problems, self-reported osteoporosis, scoliosis, smoking status, standing height, BMI, happiness, fluid intelligence score, years of education, anxiety/panic attacks, depression and Big Five personality traits (openness to experience, conscientiousness, extraversion, agreeableness, and neuroticism) (Supplementary table 4C).

We also examined for overlap between the SNPs associated with BP and eQTLs in blood ${ }^{35}$ and 44 tissues provided by the GTEx database ${ }^{36}$ (Supplementary tables 4A) using a similar 
procedure: we examined if a SNP was available for specific expression probe in the region of interest and, if positive, the HEIDI test was performed as above.

Following Bonferroni procedure, the results of the SMR test were considered statistically significant at $\mathrm{p}<3 \times 10^{-5}(0.05 / 1685$, where 1685 is the number of probes available in blood eQTL data and GTEx data for three studied loci) for eQTLs; and $\mathrm{p}<9.8 \times 10^{-4}(0.05 /(17 \times 3)$ accounting for 3 studied loci and 17 complex traits) for complex traits.

For the HEIDI tests, a hypothesis of pleiotropy was rejected at $\mathrm{p}<0.01$; the hypothesis was accepted at $\mathrm{p}>0.01$.

\section{Gene prioritization, pathway and tissue enrichment analysis}

To prioritize genes in associated regions, gene set enrichment and tissue/cell type enrichment analyses were carried out using DEPICT software v. 1 rel. $194^{73}$. For this analysis we chose independent (by COJO) variants found in the BP meta-analysis results $(\mathrm{N}=453,862)$ with $\mathrm{p}<5 \times 10^{-8}$ (23 SNPs) and $\mathrm{p}<1 \times 10^{-5}$ (227 SNPs). We used a random subset of 10,000 individuals from the UK Biobank for calculation of LD (the same subsets as used for COJO analysis).

We also conducted gene analysis and gene-set analysis using MAGMA v1.6 included in FUMA web tool ${ }^{44}$. Reference genome was 1000 genomes phase 3. The MAGMA output from FUMA was based on summary statistics. We used standard options suggested by FUMA web tool for this analysis. 


\section{REFERENCES}

1. Hoy, D. et al. The global burden of low back pain: estimates from the Global Burden of Disease 2010 study. Ann Rheum Dis 73, 968-74 (2014).

2. $\quad$ Manchikanti, L., Singh, V., Falco, F.J., Benyamin, R.M. \& Hirsch, J.A. Epidemiology of low back pain in adults. Neuromodulation 17 Suppl 2, 3-10 (2014).

3. Collaborators, G.D.a.I.I.a.P. Global, regional, and national incidence, prevalence, and years lived with disability for 328 diseases and injuries for 195 countries, 1990-2016: a systematic analysis for the Global Burden of Disease Study 2016. Lancet 390, 1211-1259 (2017).

4. Taylor, J.B., Goode, A.P., George, S.Z. \& Cook, C.E. Incidence and risk factors for first-time incident low back pain: a systematic review and meta-analysis. Spine J 14, 2299-319 (2014).

5. Zheng, C.J. \& Chen, J. Disc degeneration implies low back pain. Theor Biol Med Model 12, 24 (2015).

6. Videman, T. et al. Associations between back pain history and lumbar MRI findings. Spine (Phila Pa 1976) 28, 582-8 (2003).

7. Gore, M., Sadosky, A., Stacey, B.R., Tai, K.S. \& Leslie, D. The burden of chronic low back pain: clinical comorbidities, treatment patterns, and health care costs in usual care settings. Spine (Phila Pa 1976) 37, E668-77 (2012).

8. Maniadakis, N. \& Gray, A. The economic burden of back pain in the UK. Pain 84, 95-103 (2000).

9. Hong, J., Reed, C., Novick, D. \& Happich, M. Costs associated with treatment of chronic low back pain: an analysis of the UK General Practice Research Database. Spine (Phila Pa 1976) 38, 75-82 (2013).

10. Battie, M.C., Videman, T., Levalahti, E., Gill, K. \& Kaprio, J. Heritability of low back pain and the role of disc degeneration. Pain 131, 272-80 (2007).

11. Junqueira, D.R. et al. Heritability and lifestyle factors in chronic low back pain: results of the Australian twin low back pain study (The AUTBACK study). Eur J Pain 18, 1410-8 (2014).

12. Nyman, T., Mulder, M., Iliadou, A., Svartengren, M. \& Wiktorin, C. High heritability for concurrent low back and neck-shoulder pain: a study of twins. Spine (Phila Pa 1976) 36, E1469-76 (2011).

13. MacGregor, A.J., Andrew, T., Sambrook, P.N. \& Spector, T.D. Structural, psychological, and genetic influences on low back and neck pain: a study of adult female twins. Arthritis Rheum 51, 160-7 (2004).

14. Kalichman, L. \& Hunter, D.J. The genetics of intervertebral disc degeneration. Familial predisposition and heritability estimation. Joint Bone Spine 75, 383-7 (2008).

15. Battie, M.C., Videman, T., Levalahti, E., Gill, K. \& Kaprio, J. Genetic and environmental effects on disc degeneration by phenotype and spinal level: a multivariate twin study. Spine (Phila Pa 1976) 33, 2801-8 (2008).

16. Livshits, G. et al. Lumbar disc degeneration and genetic factors are the main risk factors for low back pain in women: the UK Twin Spine Study. Ann Rheum Dis 70, 1740-5 (2011).

17. Pinheiro, M.B. et al. Genetics and the environment affect the relationship between depression and low back pain: a co-twin control study of Spanish twins. Pain 156, 496-503 (2015).

18. Zadro, J.R. et al. Does educational attainment increase the risk of low back pain when genetics are considered? A population-based study of Spanish twins. Spine J 17, 518-530 (2017).

19. Dario, A.B. et al. The relationship between obesity, low back pain, and lumbar disc degeneration when genetics and the environment are considered: a systematic review of twin studies. Spine J 15, 1106-17 (2015).

20. Malkin, I. et al. Low back and common widespread pain share common genetic determinants. Ann Hum Genet 78, 357-66 (2014).

21. Sudlow, C. et al. UK biobank: an open access resource for identifying the causes of a wide range of complex diseases of middle and old age. PLoS Med 12, e1001779 (2015).

22. Suri, P. et al. Genome-wide Meta-analysis of 158,000 Individuals of European Ancestry Identifies Three Loci Associated with Chronic Back Pain. (2018).

23. Bjornsdottir, G. et al. Sequence variant at 8q24.21 associates with sciatica caused by lumbar disc herniation. Nat Commun 8, 14265 (2017).

24. Song, Y.Q. et al. Lumbar disc degeneration is linked to a carbohydrate sulfotransferase 3 variant. J Clin Invest 123, 4909-17 (2013).

25. Hermanns, P. et al. Congenital joint dislocations caused by carbohydrate sulfotransferase 3 deficiency in recessive Larsen syndrome and humero-spinal dysostosis. Am J Hum Genet 82, 1368-74 (2008). 
26. Thiele, H. et al. Loss of chondroitin 6-O-sulfotransferase-1 function results in severe human chondrodysplasia with progressive spinal involvement. Proc Natl Acad Sci U S A 101, 10155-60 (2004).

27. Tuysuz, B. et al. Omani-type spondyloepiphyseal dysplasia with cardiac involvement caused by a missense mutation in CHST3. Clin Genet 75, 375-83 (2009).

28. Unger, S. et al. Phenotypic features of carbohydrate sulfotransferase 3 (CHST3) deficiency in 24 patients: congenital dislocations and vertebral changes as principal diagnostic features. Am J Med Genet A 152A, 2543-9 (2010).

29. van Roij, M.H. et al. Spondyloepiphyseal dysplasia, Omani type: further definition of the phenotype. Am $J$ Med Genet A 146A, 2376-84 (2008).

30. Hadchouel, A. et al. Identification of SPOCK2 as a susceptibility gene for bronchopulmonary dysplasia. Am J Respir Crit Care Med 184, 1164-70 (2011).

31. Nordgard, S.H. et al. Genome-wide analysis identifies 16q deletion associated with survival, molecular subtypes, mRNA expression, and germline haplotypes in breast cancer patients. Genes Chromosomes Cancer 47, 680-96 (2008).

32. David, S.P. et al. Genome-wide meta-analyses of smoking behaviors in African Americans. Transl Psychiatry 2, e119 (2012).

33. Vannahme, C. et al. Molecular cloning of testican-2: defining a novel calcium-binding proteoglycan family expressed in brain. $J$ Neurochem 73, 12-20 (1999).

34. Zhu, Z. et al. Integration of summary data from GWAS and eQTL studies predicts complex trait gene targets. Nat Genet 48, 481-7 (2016).

35. Westra, H.J. et al. Systematic identification of trans eQTLs as putative drivers of known disease associations. Nat Genet 45, 1238-1243 (2013).

36. GTEx_Consortium. Human genomics. The Genotype-Tissue Expression (GTEx) pilot analysis: multitissue gene regulation in humans. Science 348, 648-60 (2015).

37. Okbay, A. et al. Genetic variants associated with subjective well-being, depressive symptoms, and neuroticism identified through genome-wide analyses. Nat Genet 48, 624-33 (2016).

38. Hammerschlag, A.R. et al. Genome-wide association analysis of insomnia complaints identifies risk genes and genetic overlap with psychiatric and metabolic traits. Nat Genet 49, 1584-1592 (2017).

39. Major Depressive Disorder Working Group of the Psychiatric, G.C. et al. A mega-analysis of genome-wide association studies for major depressive disorder. Mol Psychiatry 18, 497-511 (2013).

40. Barban, N. et al. Genome-wide analysis identifies 12 loci influencing human reproductive behavior. Nat Genet 48, 1462-1472 (2016).

41. Okbay, A. et al. Genome-wide association study identifies 74 loci associated with educational attainment. Nature 533, 539-42 (2016).

42. Pilling, L.C. et al. Human longevity is influenced by many genetic variants: evidence from 75,000 UK Biobank participants. Aging (Albany NY) 8, 547-60 (2016).

43. Rietveld, C.A. et al. GWAS of 126,559 individuals identifies genetic variants associated with educational attainment. Science 340, 1467-71 (2013).

44. Watanabe, K., Taskesen, E., van Bochoven, A. \& Posthuma, D. Functional mapping and annotation of genetic associations with FUMA. Nat Commun 8, 1826 (2017).

45. Nikolsky, Y. et al. Genome-wide functional synergy between amplified and mutated genes in human breast cancer. Cancer Res 68, 9532-40 (2008).

46. Heuch, I., Heuch, I., Hagen, K. \& Zwart, J.A. Association between body height and chronic low back pain: a follow-up in the Nord-Trondelag Health Study. BMJ Open 5, e006983 (2015).

47. MacGregor, A.J., Andrew, T., Sambrook, P.N. \& Spector, T.D. Structural, psychological, and genetic influences on low back and neck pain: a study of adult female twins. Arthritis and rheumatism 51, 160-7 (2004).

48. Taylor, J.B., Goode, A.P., George, S.Z. \& Cook, C.E. Incidence and risk factors for first-time incident low back pain: a systematic review and meta-analysis. The spine journal : official journal of the North American Spine Society 14, 2299-319 (2014).

49. Williams, F.M. et al. Novel genetic variants associated with lumbar disc degeneration in northern Europeans: a meta-analysis of 4600 subjects. Ann Rheum Dis 72, 1141-8 (2013).

50. Ferreira, P.H., Beckenkamp, P., Maher, C.G., Hopper, J.L. \& Ferreira, M.L. Nature or nurture in low back pain? Results of a systematic review of studies based on twin samples. Eur J Pain (2013). 
51. Hartvigsen, J. et al. Heritability of spinal pain and consequences of spinal pain: a comprehensive genetic epidemiologic analysis using a population-based sample of 15,328 twins ages 20-71 years. Arthritis and rheumatism 61, 1343-51 (2009).

52. Hestbaek, L., Iachine, I.A., Leboeuf-Yde, C., Kyvik, K.O. \& Manniche, C. Heredity of low back pain in a young population: a classical twin study. Twin Res 7, 16-26 (2004).

53. Junqueira, D.R. et al. Heritability and lifestyle factors in chronic low back pain: results of the Australian twin low back pain study (The AUTBACK study). European journal of pain 18, 1410-8 (2014).

54. Sambrook, P.N., MacGregor, A.J. \& Spector, T.D. Genetic influences on cervical and lumbar disc degeneration: a magnetic resonance imaging study in twins. Arthritis Rheum 42, 366-72 (1999).

55. McIntosh, A.M. et al. Genetic and Environmental Risk for Chronic Pain and the Contribution of Risk Variants for Major Depressive Disorder: A Family-Based Mixed-Model Analysis. PLoS Med 13, e1002090 (2016).

56. Gasperi, M., Herbert, M., Schur, E., Buchwald, D. \& Afari, N. Genetic and Environmental Influences on Sleep, Pain, and Depression Symptoms in a Community Sample of Twins. Psychosom Med 79, 646-654 (2017).

57. Ogata, S., Williams, F. \& Burri, A. Genetic Factors Explain the Association Between Pain Catastrophizing and Chronic Widespread Pain. J Pain 18, 1111-1116 (2017).

58. Pinheiro, M.B. et al. Genetic and Environmental Contributions to Sleep Quality and Low Back Pain: A Population-Based Twin Study. Psychosom Med 80, 263-270 (2018).

59. Henry, D.E., Chiodo, A.E. \& Yang, W. Central nervous system reorganization in a variety of chronic pain states: a review. $P M R \mathbf{3}, 1116-25$ (2011).

60. Ward, S.R. et al. Architectural analysis and intraoperative measurements demonstrate the unique design of the multifidus muscle for lumbar spine stability. J Bone Joint Surg Am 91, 176-85 (2009).

61. Ward, S.R. et al. Passive mechanical properties of the lumbar multifidus muscle support its role as a stabilizer. J Biomech 42, 1384-9 (2009).

62. Foster, N.E. et al. Prevention and treatment of low back pain: evidence, challenges, and promising directions. Lancet (2018).

63. Allegri, M. et al. Mechanisms of low back pain: a guide for diagnosis and therapy. F1000Res 5(2016).

64. Deyo, R.A. et al. Report of the NIH Task Force on Research Standards for Chronic Low Back Pain. The spine journal : official journal of the North American Spine Society 14, 1375-91 (2014).

65. Suri, P. et al. Genome-wide Meta-analysis of 158,000 Individuals of European Ancestry Identifies Three Loci Associated with Chronic Back Pain. in bioRxiv (bioRxiv).

66. Yang, J. et al. Conditional and joint multiple-SNP analysis of GWAS summary statistics identifies additional variants influencing complex traits. Nat Genet 44, 369-75, S1-3 (2012).

67. Willer, C.J., Li, Y. \& Abecasis, G.R. METAL: fast and efficient meta-analysis of genomewide association scans. Bioinformatics 26, 2190-1 (2010).

68. Zheng, J. et al. LD Hub: a centralized database and web interface to perform LD score regression that maximizes the potential of summary level GWAS data for SNP heritability and genetic correlation analysis. Bioinformatics 33, 272-279 (2017).

69. Bulik-Sullivan, B. et al. An atlas of genetic correlations across human diseases and traits. Nat Genet 47, 1236-41 (2015).

70. Devlin, B. \& Roeder, K. Genomic control for association studies. Biometrics 55, 997-1004 (1999).

71. McLaren, W. et al. The Ensembl Variant Effect Predictor. Genome Biol 17, 122 (2016).

72. Kichaev, G. et al. Integrating functional data to prioritize causal variants in statistical fine-mapping studies. PLoS Genet 10, e1004722 (2014).

73. Pers, T.H. et al. Biological interpretation of genome-wide association studies using predicted gene functions. Nat Commun 6, 5890 (2015). 


\section{SUPPLEMENTARY MATERIALS LEGEND}

Supplementary Figure 1 - QQ plot for discovery GWAS.

Supplementary Figure 2 - Manhattan plot of meta-analysis GWAS of discovery and replication samples from the UK Biobank $(\mathrm{N}=453,862)$. Red line corresponds to the genome-wide significance threshold of $5 \times 10^{-8}$, while blue line corresponds to the suggestive association threshold of $5 \times 10^{-7}$.

Supplementary Figure 3 - Barplots of genetic correlations between back pain and complex traits showing statistical significance $\left(\mathrm{p}<4.3 \times 10^{-5}\right)$.

Supplementary Figure 4 - Heatmap of partial genetic correlations for 16 traits including BP.

Supplementary Table 1 - Discovery and replication cohorts.

Supplementary Tables 2 - Results of discovery and replication GWAS.

Supplementary Table 3 - Results of COJO analysis for $\mathrm{BP}_{\mathrm{ma}}$ GWAS.

Supplementary Tables 4 - Results of SMR-HEIDI for eQTLs and seventeen risk factors.

Supplementary Table 5 - Results of PhenoScanner v1.1 for three replicated loci.

Supplementary Table 6 - Results of genetic correlations for BP (LDHub).

Supplementary Tables 7 - Results of DEPICT analysis for SNPs with $\mathrm{p}<5 \times 10^{-8}$ and $\mathrm{p}<1 \times 10^{-5}$.

Supplementary Tables 8 - Results of MAGMA analysis.

Supplementary Tables 9 - Results of VEP and PAINTOR.

Supplementary Tables 10 - Tables with correlated DHS sites for SPOCK2 and CHST3 genes.

\section{ACKNOWLEDGEMENTS}

This study was supported by the European Community's Seventh Framework Programme funded project PainOmics (Grant agreement \# 602736). The research has been conducted using the UK Biobank Resource (project \# 18219). We are grateful to the UK Biobank participants for making such research possible.

The development of software implementing SMR/HEIDI test and database for GWAS results was supported by the Russian Ministry of Science and Education under the 5-100 Excellence Program". 
Dr. Suri's time for this work was supported by VA Career Development Award \# 1IK2RX001515 from the United States (U.S.) Department of Veterans Affairs Rehabilitation Research and Development Service. Dr. Suri is a Staff Physician at the VA Puget Sound Health Care System. The contents of this work do not represent the views of the U.S. Department of Veterans Affairs or the United States Government.

Dr. Tsepilov's time for this work was supported in part by the Russian Ministry of Science and Education under the 5-100 Excellence Program.

We thank Eugene Pakhomov for developing software and database for eQTL-related analyses and Dr Sodbo Sharapov for assistance with data submission.

Cohorts for Heart and Aging Research in Genomic Epidemiology (CHARGE) Musculoskeletal Working Group: We acknowledge the following individuals from the CHARGE Musculoskeletal Working Group as non-author contributors involved in the meta-analysis of data from CHARGE cohorts : Cindy G. Boer, Michelle S. Yau, Daniel S. Evans, Andrea Gelemanovic, Traci M. Bartz, Maria Nethander, Liubov Arbeeva, Tuhina Neogi, Archie Campbell, Dan Mellstrom, Claes Ohlsson, Lynn M. Marshall, Eric Orwoll, Andre Uitterlinden, Jerome I. Rotter, Gordan Lauc, Bruce M. Psaty, Magnus K Karlsson, Nancy E. Lane, Gail Jarvik, Ozren Polasek, Marc Hochberg, Joanne M. Jordan, Joyce B. J. Van Meurs, Rebecca Jackson, Carrie M. Nielson, Braxton D. Mitchell, Blair H. Smith, Caroline Hayward, and Nicholas L. Smith.

\section{AUTHOR CONTRIBUTIONS}

MF and YT contributed to the design of the study, carried out statistical analysis, produced the figures, and first draft of the manuscript; LC provided statistical and computational support; MP and PS analysed CHARGE dataset and contributed to interpretation of the results; YA and FW conceived and oversaw the study, contributed to the design and interpretation of the results; all co-authors contributed to the final manuscript revision. 


\section{COMPETING FINANCIAL INTERESTS}

YSA and LCK are owners of Maatschap PolyOmica, a private organization, providing services, research and development in the field of computational and statistical (gen)omics. Other authors declare no conflicts of interest.

\section{DATA AVAILABILITY}

Summary statistics from our GWAS discovery and meta-analysis are available for interactive exploration at the GWAS archive (http://gwasarchive.org). The data set was also deposited at Zenodo (https://doi.org/10.5281/zenodo.1319332). The data generated in the secondary analyses of this study are included with this article in the supplementary tables. 